\title{
Antagonism of saprobe fungi from semiarid areas of the Northeast of Brazil against Sclerotinia sclerotiorum and biocontrol of soybean white mold
}

\section{Antagonismo de fungos sapróbios do semi-árido do Nordeste brasileiro contra Sclerotinia sclerotiorum e biocontrole do mofo branco da soja}

\author{
Douglas Casaroto Peitl ${ }^{1}$; Ciro Hideki Sumida ${ }^{2}$; Ricardo Marcelo Gonçalves 3 ; \\ Sérgio Florentino Pascholati ${ }^{4}$; Maria Isabel Balbi-Peña ${ }^{5 *}$
}

\section{Highlights:}

Myrothecium sp., Volutella minima, Phialomyces macrosporus and Dictyosporium tetraseriale were selected based on their antagonistic activity against Sclerotinia sclerotiorum.

Myrothecium sp. isolate 2 completely suppressed sclerotia formation and inhibited ascospore germination by over $95 \%$.

AUDPC of white mold reduced in 55.8\%, 79.7\%, and 83.2\% in soybean treated with Myrothecium sp. isolate 2, $P$. macrosporus, and $V$. minima, respectively.

\begin{abstract}
The antagonistic activity of 25 saprobe fungi from semiarid areas of the Northeast of Brazil was evaluated against Sclerotinia sclerotiorum (Lib.) de Bary (Helotiales: Sclerotiniaceae). Four fungi [Myrothecium sp. Tode (Hypocreales: Stachybotryaceae) isolate 2, Volutella minima Höhn. (Hypocreales: Nectriaceae), Phialomyces macrosporus P.C. Misra \& P.H.B. Talbot (Pezizomycotina) and Dictyosporium tetraseriale Goh, Yanna \& K.D. Hyde (Pleosporales: Dictyosporiaceae)] were selected and further tested their ability to inhibit mycelial growth, sclerotia formation and ascospore germination of S. sclerotiorum and to control white mold on soybean plants. V. minima and P. macrosporus filtrates at $50 \%$ effectively suppressed mycelial growth and Myrothecium sp. isolate 2 completely suppressed sclerotia formation and inhibited ascospore germination by over $95 \%$, the same result as commercial fungicide fluazinam. Soybean plants pre-treated with Myrothecium sp. isolate 2, P. macrosporus, and V. minima and inoculated with $S$. sclerotiorum showed a reduction of $55.8 \%, 79.7 \%$, and $83.2 \%$ of area under disease progress curve (AUDPC) of white mold, respectively, in relation to water. Collectively, these results underline the antagonistic activity of $V$. minima, P. macrosporus, and Myrothecium sp. isolate 2 against S. sclerotiorum and their potential as biocontrol agents of soybean white mold.
\end{abstract}

Key words: Biological control. Glycine max. Saprobic fungi. Sclerotinia stem rot.

\footnotetext{
${ }^{1}$ Discente do Curso de Mestrado do Programa de Pós-Graduação em Agronomia, Universidade Estadual de Londrina, UEL, Londrina, PR, Brasil. E-mail: douglas casaroto@ hotmail.com

2 Prof. Dr., Departamento de Agronomia, UEL, Londrina, PR, Brasil. E-mail: cirosumida@hotmail.com

3 Dr., Programa de Pós-Graduação em Agronomia, UEL, Londrina, PR, Brasil. E-mail: rmgoncalves@icloud.com

4 Prof. Dr., Departamento de Fitopatologia e Nematologia, Escola Superior de Agricultura "Luiz de Queiroz" Universidade de São Paulo, USP, Piracicaba, SP, Brasil. E-mail: sfpascho@usp.br

5 Prof $^{\mathrm{a}} \mathrm{Dr}^{\mathrm{a}}$, Programa de Pós-Graduação em Agronomia, UEL, Londrina, PR, Brasil. E-mail: mariabalbi@uel.br

*Author for correspondence
} 


\section{Resumo}

A atividade antagônica de 25 fungos sapróbios de região do semi-árido do Nordeste brasileiro foi avaliada contra Sclerotinia sclerotiorum (Lib.) de Bary (Helotiales: Sclerotiniaceae). Quatro fungos [Myrothecium sp. Tode (Hipocreales: Stachybotryaceae) isolate 2, Volutella minima Höhn. (Hipocreales: Nectriaceae), Phialomyces macrosporus P.C. Misra \& P.H.B. Talbot (Pezizomycotina) e Dictyosporium tetraseriale Goh, Yanna \& K.D. Hyde (Pleosporales: Dictyosporiaceae)] foram selecionados para avaliar sua capacidade de inibir o crescimento micelial, a formação de esclerócios e a germinação de ascósporos de $S$. sclerotiorum e sua eficiência de controle do mofo branco em plantas de soja. Os filtrados de V. minima e P. macrosporus a $50 \%$ de concentração suprimiram efetivamente o crescimento micelial de S. sclerotiorum. Myrothecium sp. isolado 2 suprimiu completamente a formação de escleródios e inibiu a germinação de ascósporos em mais de $95 \%$, o mesmo resultado que o fungicida comercial fluazinam. Plantas de soja pré-tratadas com Myrothecium sp. s isolado 2, P. macrosporus e V. minima e inoculados com S. sclerotiorum apresentaram redução de 55,8\%, 79,7\% e $83,2 \%$ da área abaixo da curva de progresso da doença (AACPD) mofo branco, respectivamente, em relação ao controle com água. Coletivamente, os resultados obtidos in vitro e em plantas de soja inoculadas, indicam a atividade antagônica de V. minima, P. macrosporus e Myrothecium sp. isolado 2 contra $S$. sclerotiorum e seu potencial como agentes de biocontrole do mofo branco da soja.

Palavras-chave: Controle biológico. Fungos sapróbios. Glycine max. Podridão de Sclerotinia.

\section{Introduction}

In 2018/19 Brazil was the second largest producer of soybean [Glycine max L. Merrill (Fabales: Fabaceae)] worldwide, led by the United States and followed by Argentina and China, with a total production of 117 million metric tons (United States Department of Agriculture, 2020). Despite the high productivity, more than 40 diseases can infect soybean crops in Brazil (A. M. R. Almeida et al., 2005).

White mold of soybean or Sclerotinia stem rot is caused by the fungus Sclerotinia sclerotiorum (Lib.) de Bary (Helotiales: Sclerotiniaceae) and can occur in all Brazilian regions with mild climatic conditions (southern region and cerrado highlands above $800 \mathrm{~m}$ altitude). For every increase of $10 \%$ in white mold severity, yield losses in soybean were estimated at 170 to $330 \mathrm{Kg} \mathrm{ha}^{-1}$ (Chun, Kao, Lockwood, \& Isleib, 1987; Hoffman et al., 1998; Danielson, Nelson, \& Helms, 2004).

Sclerotinia sclerotiorum is considered a polyphagous soil fungus, that infects more than 400 species of plant (Boland \& Hall, 1994). This fungus forms resistance structures (sclerotia) that can survive for long periods in the soil. Temperature conditions for sclerotia germination are affected by light intensity and moisture level. At light intensities of 80 to $90 \mathrm{~mol} \mathrm{~m} \mathrm{~m}^{-2} \mathrm{~s}^{-1}$, the optimal temperature varied from 12 to $18{ }^{\circ} \mathrm{C}$, independent of moisture level, while at light intensity of 120 to $130 \mathrm{~mol} \mathrm{~m}^{-2} \mathrm{~s}^{-1}$, the optimal temperature was 20 ${ }^{\circ} \mathrm{C}$ when the soil moisture was high (Sun \& Yang, 2000). Sclerotia germinate by forming small, cup-shaped, stalked apothecia where ascospores are formed, or by forming mycelia. Ascospores released from apothecia are carried by the wind and are responsible for plant infections (Dhingra, Mendonça, \& Macedo, 2009). Soybean plants are more susceptible to infection from blossom (R1 stage) to seed (R5 stage) (Danielson et al., 2004). Control of soybean white mold is complex due to the long susceptibility stage of the host, lack of genetic resistance and the difficulty of synchronizing fungicide sprayings with ascospore release (Morton \& Hall, 1989; Bardin \& Huang, 2001). Therefore, the utilization of alternative methods, such as biological control, becomes necessary. 
Microorganisms reported to colonize sclerotia or with antagonistic properties against Sclerotinia spp. include bacteria, mainly in the genus Bacillus (Zeng,Wang, Kirk, \& Hao, 2012; Kamal, Lindbeck, Savocchia, \& Ash, 2015; Vinodkumar, Nakkeeran, Renukadevi, \& Malathi, 2017). Nevertheless, much research has been focused on species of antagonistic or mycoparasitic fungi, including Clonostachys rosea (Preuss) Mussat (Hypocreales: Bionectriaceae) (Rabeendran, Jones, Moot, \& Stewart, 2006), Dictyosporium elegans (Corda) (Pleosporales: Dictyosporiaceae) (McCredie \& Sivasithamparam, 1985), and several species of Gliocladium and Trichoderma (Geraldine et al., 2013; Zhang et al., 2016; Elias, Domingues, Moura, Harakava, \& Patricio, 2016; Sumida et al., 2018), Paraphaeosphaeria minitans (Campb.) Verkley, Göker, Stielow (Pleosporales: Didymosphaeriaceae) (formerly Coniothyrium minitans) (Whipps, Sreenivasaprasad, Muthumeenakshi, Rogers, \& Challen, 2008; Zeng et al., 2012; Nicot et al., 2019), and Paecilomyces lilacinus (Thom) Samson (Eurotiales: Aspergillaceae) (Yang, Abdelnabby, \& Xiao, 2015).

Fungal biodiversity has been prospected in semiarid areas and various saprobe fungi were identified in the litter of the Caatinga forest in Northeast Brazil (Barbosa \& Gusmão, 2011; D. A. C. Almeida, Izabel, \& Gusmão, 2011; LeãoFerreira, Pascholati, Gusmão, \& Castañeda Ruiz, 2013; Santa Izabel \& Gusmão, 2018). Some of these saprobes were being used as potential biological control agents and resistance inducers (Resende, Milagres, Rezende, Aucique-Perez, \& Rodrigues, 2015; Barros, Fonseca, Balbi-Peña, Pascholati, \& Peitl, 2015; Rodríguez et al., 2016; Peitl et al., 2017; Ribeiro et al., 2018; Botrel et al., 2018).

In this context, the main goal of the present study was to select saprobe fungi from semiarid areas of Northeast Brazil that could control white mold of soybean, which was investigated at in vitro and in vivo experiments.

\section{Materials and Methods}

Saprobe fungi and Sclerotinia sclerotiorum isolates

Saprobe fungi were isolated from the litter of the Caatinga forest (semiarid) in Northeast Brazil and are deposited in the "Coleção de Culturas de Microrganismos da Bahia (CCMB)" at the "Universidade Estadual de Feira de Santana," Bahia State, Brazil (Ministry of the Environment MMA/ SISGEN code AB513B8). Species were identified by comparing their reproductive structures with the descriptions in the specialized literature (D. A. C. Almeida et al., 2011). The 25 isolates used in this work (Table 1) were cultured on potato-dextroseagar (PDA) (200 $\mathrm{g} \mathrm{L}^{-1}$ potato infusion, $20 \mathrm{~g} \mathrm{~L}^{-1}$ dextrose, $15 \mathrm{~g} \mathrm{~L}^{-1}$ agar, $\mathrm{pH} 5.6 \pm 0.2$ ) plates at 25 $\pm 2{ }^{\circ} \mathrm{C}$ and $12 / 12 \mathrm{~h}$ photoperiod. Sclerotia of $S$. sclerotiorum, were isolated from naturally infected soybean plants from Londrina, Paraná State, Brazil. Sclerotia were collected and superficially disinfected by immersion into a $70 \%$ alcohol solution for three min, then by immersion into a $0.2 \%$ sodium hypochlorite solution for three min and rinsed three times with distilled and sterilized water. Sclerotia were dried for $12 \mathrm{~h}$ in a laminar flow chamber and then transferred to PDA plates and incubated at $20^{\circ} \mathrm{C}$ in the dark for 4 days. After germination, mycelia were inoculated on PDA plates and incubated under the same conditions described above.

\section{Saprobe fungi filtrates}

Two $5 \mathrm{~mm}$ diameter mycelial disks from the edge of the saprobe fungi colonies were transferred to Erlenmeyer flasks containing $100 \mathrm{~mL}$ of potatodextrose (PD) culture broth $\left(200 \mathrm{~g} \mathrm{~L}^{-1}\right.$ potato infusion, $20 \mathrm{~g} \mathrm{~L}^{-1}$ dextrose, $\left.\mathrm{pH} 5.6 \pm 0.2\right)$. Cultures were incubated at $25 \pm 2{ }^{\circ} \mathrm{C}$ and $12 / 12 \mathrm{~h}$ photoperiod on an orbital shaker (100 rpm) for 10 days. Afterward, cultures were transferred at $5 \pm 2{ }^{\circ} \mathrm{C}$ for $48 \mathrm{~h}$. The supernatant was filtrated, centrifuged twice at 5000 rpm for $15 \mathrm{~min}$ each for fungal cell removal, and stored at $5{ }^{\circ} \mathrm{C}$. 
Screening of saprobe fungi for its toxicity against Sclerotinia sclerotiorum

Filtrates from 25 saprobe fungi cultures were incorporated at $5 \%$ and $50 \%(\mathrm{v} / \mathrm{v})$ into PDA culture medium at $50{ }^{\circ} \mathrm{C}$, according to the methodology used by Sarma, Ameer Basha, Singh and Singh (2007). Five $\mathrm{mm}$ diameter mycelial disks from 3-day-old cultures of $S$. sclerotiorum grown on PDA, were transferred to the center of PDA plates with the incorporated filtrates. As a control, $S$. sclerotiorum was transferred to PDA plates without fungal filtrates. Plates were incubated at $20 \pm 2$ ${ }^{\circ} \mathrm{C}$ and $12 / 12 \mathrm{~h}$ photoperiod. Colony size was assessed daily by two orthogonal measurements of the diameter of the fungal colony until the control treatment reached the entire plate. Daily diameter measurements were used to determine the mycelial growth rate (MGR) (Oliveira, 1991):

$$
M G R=\sum_{i=1}^{n}\left(\frac{y_{i}-y_{i-1}}{n}\right)
$$

Where, $\mathrm{y}$ is the diameter $(\mathrm{cm})$ at each measurement at the $i^{\text {th }}$ observation and $n$ is the number of days after inoculation.

After 21 days, the number and weight of sclerotia formed on the plates were assessed.

Effect of saprobe fungal filtrates on Sclerotinia sclerotiorum mycelial growth and sclerotia formation

Based on the previous screening, Myrothecium sp. Tode (Hypocreales: Stachybotryaceae) isolate 2, Dictyosporium tetraseriale Goh, Yanna \& K.D. Hyde (Pleosporales: Dictyosporiaceae), Volutella minima Höhn. (Hypocreales: Nectriaceae) and Phialomyces macrosporus P.C. Misra \& P.H.B. Talbot (Pezizomycotina) filtrates were incorporated at $5 \%$ and $50 \%(\mathrm{v} / \mathrm{v})$ in PDA medium at $50{ }^{\circ} \mathrm{C}$. Five $\mathrm{mm}$ diameter mycelial disks from 3-day-old cultures of S. sclerotiorum grown on PDA, were transferred to the center of PDA plates with the incorporated filtrates and incubated at $20 \pm 2{ }^{\circ} \mathrm{C}$ and
12/12 h photoperiod for 21 days. As a control, $S$. sclerotiorum was transferred on PDA plates without fungal filtrates.

Mycelial growth rate, number, and weight of sclerotia were determined as described above.

Effect of saprobe fungal filtrates on the germination of Sclerotinia sclerotiorum ascospores

Fungal filtrates were used at a concentration of $50 \%(\mathrm{v} / \mathrm{v})$ and commercial fungicide Fluazinam at $0.5 \%$ in sterile water. As a control treatment, sterile water was used.

For ascospore production, sclerotia were placed in clear polystyrene boxes $(11 \times 11 \times 3.5 \mathrm{~cm})$ with moistened autoclaved soil (eutrophic red latosol) and incubated at $20 \pm 2{ }^{\circ} \mathrm{C}$ and $12 / 12 \mathrm{~h}$ photoperiod for 45 days. Apothecia were cut and homogenized with mortar in $10 \mathrm{ml}$ of sterilized distilled water (Tolêdo-Souza \& Costa, 2007).

Aliquots of $50 \mu \mathrm{L}$ of fungal filtrates were placed in ELISA plate cells with $50 \mu \mathrm{L}$ of the ascospore suspension $\left(2 \times 10^{5}\right.$ ascospores $\left.\mathrm{mL}^{-1}\right)$. Samples were homogenized and then transferred to water-agar plates ( $15 \mathrm{~g} \mathrm{~L}^{-1}$ of agar). Plates were incubated at $20 \pm 2{ }^{\circ} \mathrm{C}$ in continuous light for $6 \mathrm{~h}$. Lactophenolcotton blue was used to stop the germination and to stain the structures. Ascospores were considered germinated when the length of their germ tube was equal to or greater than their diameter. One hundred ascospores per plate (replication) were assessed.

Effect of volatiles from saprobe fungi on Sclerotinia sclerotiorum mycelial growth and sclerotia formation

The assay was performed in two-section polystyrene Petri plates. Saprobe fungi were cultivated on PDA on one side of the plate and incubated at $25 \pm 2{ }^{\circ} \mathrm{C}$ and $12 / 12 \mathrm{~h}$ photoperiod for 7 days. Afterward, $5 \mathrm{~mm}$ diameter mycelial disks of $S$. sclerotiorum grown on PDA, were transferred to the opposite section of the plate. In the case of $P$. 
macrosporus, S. sclerotiorum was transferred only 3 days after because this fungus has faster growth than the others. Plates were kept at $25 \pm 2{ }^{\circ} \mathrm{C}$ and $12 / 12$ h photoperiod until $S$. sclerotiorum occupied the entire section of the control plates (without saprobe). Colony diameters were measured daily to obtain the MGR. The number and weight of sclerotia were assessed 21 days after inoculation.

Effect of volatiles from saprobe fungi on the germination of Sclerotinia sclerotiorum ascospores

Saprobe fungi were cultivated as described before. Water-agar was poured in the opposite section of the Petri plates, where a $50 \mu \mathrm{L}$-aliquot of ascospore suspension $\left(2 \times 10^{5}\right.$ ascospores $\left./ \mathrm{mL}\right)$ was transferred. Plates were incubated at $25^{\circ} \mathrm{C}$ in continuous light for $6 \mathrm{~h}$. Germination was stopped by lactophenol-cotton blue addition. Two hundred ascospores per plate (replication) were assessed.

Control of white mold of soybean under greenhouse conditions

Soybean seeds (cv. BMX Potencia RR) were sown in one-liter pots containing soil (eutrophic red latosol) and sand $(1: 1 \mathrm{v} / \mathrm{v})$. Saprobe fungi were cultivated in $100 \mathrm{~mL}$ PD broth $\left(200 \mathrm{~g} \mathrm{~L}^{-1}\right.$ potato infusion, $20 \mathrm{~g} \mathrm{~L}^{-1}$ dextrose, $\mathrm{pH} 5.6 \pm 0.2$ ) and incubated at $25 \pm 2{ }^{\circ} \mathrm{C}$ and $12 / 12 \mathrm{~h}$ photoperiod for 10 days. Afterwards, $100 \mathrm{~mL}$ of distilled and sterilized water was added to the cultures and homogenized. These homogenates were sprayed on soybean plants at V4 stage (four fully expanded trifoliate leaves). As a negative control, soybean plants were sprayed with distilled and sterilized water and, as a positive control, plants were sprayed with commercial inducer acibenzolar-S-methyl (ASM) $\left(30 \mathrm{~g} \mathrm{ha}^{-1}\right)$.

Three days after saprobe fungi, ASM or water sprays, soybean plants were inoculated with $S$. sclerotiorum according to the method of Kull et al. (2003), with modifications. For inoculation, a $200 \mu \mathrm{L}$ micropipette tip was used to remove mycelial disks from 3-day-old colonies of $S$. sclerotiorum grown on
$\operatorname{PDA}\left(20 \pm 2{ }^{\circ} \mathrm{C}\right.$ and $12 / 12 \mathrm{~h}$ photoperiod $)$. These tips containing mycelia were placed on the cut stems of the soybean plants. The stems were previously cut $0.5 \mathrm{~cm}$ above the last fully expanded leaf insertion ( $4^{\text {th }}$ trifoliate leaf) with sterilized scissors. After inoculation, soybean plants were kept at $20 \pm 2$ ${ }^{\circ} \mathrm{C}$ and $12 / 12 \mathrm{~h}$ photoperiod in a growth chamber. Lesions lengths were measured weekly, from the cut end of the stem to the end of the lesion, for 21 days. The AUDPC was calculated according to the formula by Shaner and Finney (1977) and normalized by dividing the AUDPC value by the total time (number of days from the first occurrence of the disease to the end of the observation period) (Fry, 1978).

\section{Experimental design and data analysis}

The in vitro experiments were arranged in a completely randomized design with five replications. The in vivo experiment was arranged in a randomized complete block design with five replications and each experimental unit corresponded to a plastic pot with a single soybean plant. The experiments were repeated.

Data were submitted to analysis of variance at 0.05 level of significance. When treatment effects were significant, means were compared by the Scott-Knott test $(\mathrm{p} \leq 0.05)$ in the screening test and by Tukey test $(\mathrm{p} \leq 0.05)$ in tests with the four selected saprobes.

\section{Results}

Screening of saprobe fungi

Curvularia eragrostidis (Henn.) Meyer (Pleosporales: Pleosporaceae), Gonytrichum macrocladum (Sacc.) Hughes (Chaetosphaeriales: Chaetosphaeriaceae), Myrothecium sp. isolate 2, Phialomyces macrosporus, Stachybotrys globosa Misra, Srivast (Hypocreales: Stachybotryaceae) and Volutella minima filtrates at $5 \%(\mathrm{v} / \mathrm{v})$, reduced the MGR of S. sclerotiorum. Phialomyces macrosporus showed the lowest rate $(0.67 \mathrm{~cm} /$ day $)$, which was 
statistically different from the control treatment and from the other saprobe filtrates (Table 1). At the concentration of $50 \%$, P. macrosporus and V. minima filtrates completely inhibited the mycelial growth of S. sclerotiorum. At the same concentration, Beltraniella portoricensis (Stevens) Piroz., Patil (Amphisphaeriales: Amphisphaeriaceae), Clonostachys rosea, Dictyosporium tetraseriale, Myrothecium sp. Isolate 2, and Sarcopodium circinatum Ehrenb. (Pezizomycotina) filtrates reduced the mycelial growth in about $76 \%$.
Sclerotia number and weight exhibited greater variationthanmycelial growth(Table 1).Stachybotrys chartarum (Ehrenb.) Hughes (Hypocreales: Stachybotryaceae) filtrates, completely inhibited sclerotia formation at both concentrations. At $50 \%$, B. portoricensis, Dictyochaeta heteroderae (Morgan-Jones) Carris, Glawe, (Chaetosphaeriales: Chaetosphaeriaceae) and Myrothecium sp. isolate 2 filtrates also completely inhibited sclerotia formation. Because P. macrosporus formed so few sclerotia, their weight was almost zero.

Table 1

Mycelial growth rate (MGR), number and weight of sclerotia of Sclerotinia sclerotiorum in potato dextrose agar culture medium amended with saprobe fungal filtrates at $5 \%$ and $50 \%(\mathrm{v} / \mathrm{v})$

\begin{tabular}{|c|c|c|c|c|c|c|}
\hline \multirow{3}{*}{ Treatments } & \multirow{2}{*}{\multicolumn{2}{|c|}{$\operatorname{MGR}^{\text {ab }}$ (cm/day) }} & \multicolumn{4}{|c|}{ Sclerotia $^{a}$} \\
\hline & & & \multicolumn{2}{|c|}{ Number } & \multicolumn{2}{|c|}{ Weight (g) } \\
\hline & $5 \%$ & $50 \%$ & $5 \%$ & $50 \%$ & $5 \%$ & $\mathbf{5 0 \%}$ \\
\hline Control treatment & $2.45 \pm 0.04 a$ & $2.46 \pm 0.03 \mathrm{a}$ & $26.4 \pm 4,27 \mathrm{a}$ & $21.6 \pm 2.98 b$ & $0.25 \pm 0.009 \mathrm{a}$ & $0.30 \pm 0.023 b$ \\
\hline Beltrania copaifera & $2.42 \pm 0.26 \mathrm{a}$ & $2.62 \pm 0.03 \mathrm{a}$ & $13.0 \pm 1.92 \mathrm{a}$ & $3.40 \pm 0.51 \mathrm{~d}$ & $0.13 \pm 0.020 b$ & $0.08 \pm 0.008 \mathrm{~d}$ \\
\hline Beltrania rhombica & $2.52 \pm 0.01 \mathrm{a}$ & $2.44 \pm 0.06 \mathrm{a}$ & $9.80 \pm 3.10 b$ & $8.20 \pm 1.50 \mathrm{~d}$ & $0.31 \pm 0.162 \mathrm{a}$ & $0.21 \pm 0.034 \mathrm{c}$ \\
\hline Beltraniella portoricensis & $2.41 \pm 0.04 \mathrm{a}$ & $0.77 \pm 0.08 \mathrm{~d}$ & $6.60 \pm 1.83 c$ & $0.00 \pm 0.00 \mathrm{~d}$ & $0.18 \pm 0.012 \mathrm{a}$ & $0.00 \pm 0.000 \mathrm{e}$ \\
\hline Chloridium virescens var. virescens & $2.46 \pm 0.03 \mathrm{a}$ & $1.24 \pm 0.08 \mathrm{c}$ & $12.0 \pm 2.19 b$ & $6.00 \pm 2.51 \mathrm{~d}$ & $0.13 \pm 0.028 b$ & $0.06 \pm 0.008 \mathrm{~d}$ \\
\hline Clonostachys rosea & $2.56 \pm 0.06 \mathrm{a}$ & $0.47 \pm 0.10 \mathrm{~d}$ & $10.8 \pm 3.07 \mathrm{~b}$ & $18.4 \pm 1.29 \mathrm{c}$ & $0.12 \pm 0.024 b$ & $0.17 \pm 0.015 \mathrm{c}$ \\
\hline Curvularia eragrostidis & $1.87 \pm 0.11 b$ & $2.32 \pm 0.10 b$ & $5.00 \pm 1.30 \mathrm{c}$ & $2.60 \pm 2.14 d$ & $0.08 \pm 0.021 b$ & $0.02 \pm 0.009 \mathrm{e}$ \\
\hline Curvularia inaequalis & $2.42 \pm 0.04 \mathrm{a}$ & $2.20 \pm 0.04 b$ & $14.4 \pm 2.14 b$ & $14.4 \pm 0.68 \mathrm{c}$ & $0.14 \pm 0.012 b$ & $0.13 \pm 0.011 \mathrm{~d}$ \\
\hline Dictyochaeta heteroderae & $2.60 \pm 0.11 \mathrm{a}$ & $1.97 \pm 0.09 b$ & $10.6 \pm 3.31 b$ & $0.00 \pm 0.00 \mathrm{~d}$ & $0.13 \pm 0.027 b$ & $0.00 \pm 0.000 \mathrm{e}$ \\
\hline Dictyosporium tetraseriale & $2.47 \pm 0.13 \mathrm{a}$ & $0.52 \pm 0.09 \mathrm{~d}$ & $4.60 \pm 0.68 \mathrm{c}$ & $3.60 \pm 0.81 \mathrm{~d}$ & $0.08 \pm 0.011 b$ & $0.05 \pm 0.005 \mathrm{~d}$ \\
\hline Gonytrichum chlamydosporium & $2.31 \pm 0.08 \mathrm{a}$ & $1.37 \pm 0.35 \mathrm{c}$ & $7.20 \pm 3.38 \mathrm{c}$ & $3.00 \pm 1.26 \mathrm{~d}$ & $0.03 \pm 0.014 b$ & $0.03 \pm 0.006 \mathrm{e}$ \\
\hline Gonytrichum macrocladum & $2.05 \pm 2.05 b$ & $1.80 \pm 0.23 b$ & $10.2 \pm 2.78 b$ & $3.25 \pm 1.49 \mathrm{~d}$ & $0.08 \pm 0.018 b$ & $0.05 \pm 0.017 \mathrm{~d}$ \\
\hline Lappodechium lageniforme & $2.77 \pm 0.06 \mathrm{a}$ & $2.69 \pm 0.13 \mathrm{a}$ & $9.80 \pm 1.83 b$ & $26.8 \pm 3.85 \mathrm{a}$ & $0.20 \pm 0.033 a$ & $0.41 \pm 0.043 a$ \\
\hline Memnoniella echinata & $2.58 \pm 0.05 a$ & $2.64 \pm 0.07 \mathrm{a}$ & $15.4 \pm 2.56 b$ & $20.0 \pm 5.10 b$ & $0.13 \pm 0.011 b$ & $0.13 \pm 0.032 \mathrm{~d}$ \\
\hline Myrothecium sp. isolate 1 & $2.55 \pm 0.04 \mathrm{a}$ & $2.62 \pm 0.03 \mathrm{a}$ & $12.6 \pm 1.21 b$ & $17.8 \pm 4.76 \mathrm{c}$ & $0.15 \pm 0.019 a$ & $0.26 \pm 0.006 \mathrm{~d}$ \\
\hline Myrothecium sp. isolate 2 & $1.85 \pm 0.12 b$ & $0.41 \pm 0.41 \mathrm{~d}$ & $10.6 \pm 2.92 b$ & $0.00 \pm 0.00 \mathrm{~d}$ & $0.22 \pm 0.054 \mathrm{a}$ & $0.00 \pm 0.000 \mathrm{e}$ \\
\hline Periconia hispidula & $2.73 \pm 0.07 \mathrm{a}$ & $2.18 \pm 0.31 b$ & $5.40 \pm 1.81 \mathrm{c}$ & $2.80 \pm 0.37 \mathrm{~d}$ & $0.06 \pm 0.011 b$ & $0.06 \pm 0.009 \mathrm{~d}$ \\
\hline Phialomyces macrosporus & $0.67 \pm 0.17 \mathrm{c}$ & $0.00 \pm 0.00 \mathrm{e}$ & $4.20 \pm 1.11 \mathrm{c}$ & $0.40 \pm 0.40 \mathrm{~d}$ & $0.09 \pm 0.022 b$ & $0.00 \pm 0.000 \mathrm{e}$ \\
\hline Pithomyces chartarum & $2.35 \pm 0.09 \mathrm{a}$ & $1.07 \pm 0.10 \mathrm{c}$ & $9.80 \pm 2.60 b$ & $6.60 \pm 0.68 \mathrm{~d}$ & $0.10 \pm 0.019 b$ & $0.07 \pm 0.009 \mathrm{~d}$ \\
\hline Pseudobotrytis terrestris & $2.34 \pm 0.01 \mathrm{a}$ & $1.49 \pm 0.08 \mathrm{c}$ & $8.00 \pm 2.17 \mathrm{c}$ & $3.20 \pm 1.02 \mathrm{~d}$ & $0.17 \pm 0.054 a$ & $0.07 \pm 0.006 \mathrm{~d}$ \\
\hline Sarcopodium circinatum & $2.51 \pm 0.03 \mathrm{a}$ & $0.73 \pm 0.02 \mathrm{~d}$ & $10.4 \pm 3.67 b$ & $12.4 \pm 2.38 \mathrm{c}$ & $0.17 \pm 0.020 \mathrm{a}$ & $0.07 \pm 0.041 \mathrm{~d}$ \\
\hline Stachybotrys chartarum & $2.48 \pm 0.03 \mathrm{a}$ & $2.66 \pm 0.03 \mathrm{a}$ & $0.00 \pm 0.00 \mathrm{c}$ & $0.00 \pm 0.00 \mathrm{~d}$ & $0.00 \pm 0.000 \mathrm{~b}$ & $0.00 \pm 0.000 \mathrm{e}$ \\
\hline Stachybotrys globosa & $2.20 \pm 0.28 b$ & $2.27 \pm 0.40 b$ & $11.8 \pm 3.32 b$ & $14.2 \pm 2.65 \mathrm{c}$ & $0.21 \pm 0.053 \mathrm{a}$ & $0.40 \pm 0.103 \mathrm{a}$ \\
\hline Stachylidium bicolor & $2.53 \pm 0.03 a$ & $1.99 \pm 0.07 b$ & $13.4 \pm 1.36 b$ & $2.00 \pm 1.26 \mathrm{~d}$ & $0.18 \pm 0.031 \mathrm{a}$ & $0.04 \pm 0.025 \mathrm{e}$ \\
\hline Volutella minima & $2.12 \pm 0.44 b$ & $0.00 \pm 0.00 \mathrm{e}$ & $3.80 \pm 1.39 \mathrm{c}$ & $6.00 \pm 1.58 \mathrm{~d}$ & $0.08 \pm 0.015 b$ & $0.08 \pm 0.014 \mathrm{~d}$ \\
\hline Zygosporium echinosporum & $2.32 \pm 0.04 \mathrm{a}$ & $2.22 \pm 0.03 b$ & $10.2 \pm 0.58 b$ & $6.40 \pm 1.29 \mathrm{~d}$ & $0.22 \pm 0.004 \mathrm{a}$ & $0.18 \pm 0.023 \mathrm{c}$ \\
\hline
\end{tabular}

${ }^{a}$ Mean \pm SE $(n=5$ replicates) within each column followed by the same letter are not significantly different $(\mathrm{P}<0.05)$ according to Scott-Knott test. ${ }^{\mathrm{b}} \mathrm{MGR}=$ (current colony diameter - colony diameter of the previous day $) /($ number of days after inoculation). 
Table 2

Mycelial growth rate (MGR), number and weight of sclerotia of Sclerotinia sclerotiorum cultured in potato dextrose agar culture medium amended with saprobe fungal filtrates at $5 \%$ and $50 \%$ (v/v)

\begin{tabular}{|c|c|c|c|c|c|c|}
\hline \multirow{3}{*}{ Treatments } & \multirow{2}{*}{\multicolumn{2}{|c|}{$\operatorname{MGR}(\mathrm{cm} / \mathrm{day})^{\mathrm{c}}$}} & \multicolumn{4}{|c|}{ Sclerotia } \\
\hline & & & \multicolumn{2}{|c|}{ Number } & \multicolumn{2}{|c|}{ Weight (g) } \\
\hline & $5 \%$ & $50 \%$ & $5 \%$ & $50 \%$ & $5 \%$ & $50 \%$ \\
\hline Control treatment ${ }^{\mathrm{a}}$ & $2.5 \pm 0.08 \mathrm{a}$ & $2.4 \pm 0.08 \mathrm{a}$ & $26.4 \pm 6.08 \mathrm{a}$ & $21.6 \pm 5.25 \mathrm{a}$ & $0.25 \pm 0.07 \mathrm{a}$ & $0.30 \pm 0.09 a$ \\
\hline Dictyosporium tetraseriale & $2.4 \pm 0.08 \mathrm{a}$ & $0.5 \pm 0.02 b$ & $5.0 \pm 1.15 b$ & $3.6 \pm 0.87 \mathrm{~b}$ & $0.08 \pm 0.02 b$ & $0.05 \pm 0.01 \mathrm{~b}$ \\
\hline Myrothecium sp. isolate 2 & $1.8 \pm 0.06 \mathrm{a}$ & $0.4 \pm 0.01 \mathrm{~b}$ & $10.6 \pm 2.44 b$ & $0.0 \pm 0.00 \mathrm{~b}$ & $0.22 \pm 0.06 \mathrm{a}$ & $0.0 \pm 0.00 \mathrm{~b}$ \\
\hline Phialomyces macrosporus & $0.7 \pm 0.02 b$ & $0.0 \pm 0.00 \mathrm{c}$ & $3.7 \pm 0.85 b$ & $0.5 \pm 0.12 b$ & $0.05 \pm 0.01 \mathrm{~b}$ & $0.01 \pm 0.00 \mathrm{~b}$ \\
\hline Volutella minima & $2.1 \pm 0.07 \mathrm{a}$ & $0.0 \pm 0.00 \mathrm{c}$ & $3.8 \pm 0.88 \mathrm{~b}$ & $6.0 \pm 1.46 \mathrm{~b}$ & $0.08 \pm 0.02 b$ & $0.08 \pm 0.02 b$ \\
\hline Control treatment ${ }^{\mathrm{b}}$ & $2.4 \pm 0.08 \mathrm{a}$ & $2.4 \pm 0.09 \mathrm{a}$ & $28.7 \pm 6.47 \mathrm{a}$ & $19.8 \pm 5.06 \mathrm{a}$ & $0.30 \pm 0.08 \mathrm{a}$ & $0.30 \pm 0.08 \mathrm{a}$ \\
\hline Dictyosporium tetraseriale & $2.5 \pm 0.08 \mathrm{a}$ & $0.8 \pm 0.03 b$ & $5.0 \pm 1.13 b$ & $2.3 \pm 0.59 b$ & $0.09 \pm 0.02 b$ & $0.05 \pm 0.01 b$ \\
\hline Myrothecium sp. isolate 2 & $1.9 \pm 0.06 \mathrm{a}$ & $0.4 \pm 0.01 b$ & $12.3 \pm 2.77 b$ & $0.0 \pm 0.00 \mathrm{~b}$ & $0.25 \pm 0.07 \mathrm{a}$ & $0.0 \pm 0.00 \mathrm{~b}$ \\
\hline Phialomyces macrosporus & $0.7 \pm 0.02 b$ & $0.0 \pm 0.00 \mathrm{c}$ & $3.7 \pm 0.83 b$ & $0.5 \pm 0.13 b$ & $0.05 \pm 0.01 \mathrm{~b}$ & $0.01 \pm 0.00 \mathrm{~b}$ \\
\hline Volutella minima & $2.1 \pm 0.07 \mathrm{a}$ & $0.0 \pm 0.00 \mathrm{c}$ & $2.9 \pm 0.65 b$ & $5.4 \pm 1.38 b$ & $0.08 \pm 0.02 b$ & $0.08 \pm 0.02 b$ \\
\hline
\end{tabular}

${ }^{a}$ First experiment, mean \pm SE $(n=5$ replicates) within each column followed by the same letter are not significantly different $(\mathrm{P}<0.05)$ according to Tukey test.

${ }^{b}$ Second experiment, mean \pm SE ( $n=5$ replicates) within each column followed by the same letter are not significantly different $(\mathrm{P}<0.05)$ according to Tukey test.

${ }^{\mathrm{c}} \mathrm{MGR}=($ current colony diameter - colony diameter of the previous day)/(number of days after inoculation).

Effect of saprobe filtrates on mycelial growth, sclerotia formation and germination of ascospores of Sclerotinia sclerotiorum

When saprobe filtrates were assessed at 5\%, only P. macrosporus reduced MGR in both experiments (Table 2). Regarding sclerotia formation, it was observed that all treatments were different from the control, reducing sclerotia number in both experiments. Considering the effect on sclerotia weight, only Myrothecium sp. isolate 2 filtrates did not reduce this variable in both experiments.

When fungal filtrates were assessed at 50\%, all treatments reduced $S$. sclerotiorum MGR. Myrothecium sp. isolate 2 and D. tetraseriale filtrates showed MGR means of 0.4 and $0.65 \mathrm{~cm} /$ day, respectively. Volutella minima and P. macrosporus filtrates completely inhibited $S$. sclerotiorum mycelial growth in both experiments. All treatments reduced the number and weight of sclerotia formed. Myrothecium sp. isolate 2 filtrates completely inhibited the formation of these structures.

Treatments with fluazinam and Myrothecium sp. isolate 2 reduced the ascospore germination (Table 3) in about $90.7 \%$ and $97 \%$, respectively (average from both experiments). D. tetraseriale and $V$. minima filtrates reduced ascospore germination in about $23.9 \%$ and $30.8 \%$, respectively (average from both experiments). Treatment with P. macrosporus filtrate did not significantly reduce ascospore germination. 


\section{Table 3}

Effect of saprobe filtrates and fluazinam on the germination of ascospores of Sclerotinia sclerotiorum

\begin{tabular}{lccc}
\hline Treatments & \multicolumn{2}{c}{ Germination (\%) } & Germination inhibition (\%) \\
\hline Control treatment $^{\mathrm{a}}$ & $92.8 \pm 2.66$ & $\mathrm{a}^{1}$ & - \\
Dictyosporium tetraseriale & $70.2 \pm 2.01$ & $\mathrm{~b}$ & 24.4 \\
Fluazinam & $8.80 \pm 0.25$ & $\mathrm{c}$ & 90.5 \\
Myrothecium sp. isolate 2 & $2.60 \pm 0.07$ & $\mathrm{c}$ & 97.2 \\
Phialomyces macrosporus & $89.0 \pm 2.55$ & $\mathrm{a}$ & 4.10 \\
Volutella minima & $65.2 \pm 1.87$ & $\mathrm{~b}$ & 29.7 \\
\hline Control treatment & $94.2 \pm 2.86$ & $\mathrm{a}$ & - \\
Dictyosporium tetraseriale & $72.3 \pm 2.20$ & $\mathrm{~b}$ & 23.3 \\
Fluazinam & $8.70 \pm 0.26$ & $\mathrm{c}$ & 90.8 \\
Myrothecium sp. isolate 2 & $3.10 \pm 0.09$ & $\mathrm{c}$ & 96.7 \\
Phialomyces macrosporus & $89.3 \pm 2.72$ & $\mathrm{a}$ & 5.20 \\
Volutella minima & $64.2 \pm 1.95$ & $\mathrm{~b}$ & 31.9 \\
\hline
\end{tabular}

${ }^{a}$ First experiment, mean \pm SE ( $n=5$ replicates) within each column followed by the same letter are not significantly different $(\mathrm{P}<0.05)$ according to Tukey test.

${ }^{b}$ Second experiment, mean \pm SE ( $n=5$ replicates) within each column followed by the same letter are not significantly different $(\mathrm{P}<0.05)$ according to Tukey test.

Effect of volatiles from saprobe fungi on mycelial growth, sclerotia formation and ascospore germination of Sclerotinia sclerotiorum

Only $P$. macrosporus volatiles inhibited the mycelial growth of $S$. sclerotiorum in about $60 \%$, MGR in approximately 54\% (Table 4) and ascospore germination in approximately 30\% (Table 5). Regarding sclerotia number, treatments with $D$. tetraseriale, P. macrosporus, and V. minima volatiles reduced sclerotia formation. Sclerotia weight was not affected by fungal volatiles.
Control of soybean white mold under greenhouse conditions

Lesion length of white mold at 7 days after inoculation (DAI) in soybean plants treated with ASM and D. tetraseriale was not different from that in untreated and inoculated plants (Table 6). Plants treated with Myrothecium sp. isolate 2 and P. macrosporus showed shorter lesions $(0.8 \mathrm{~cm})$ than ASM and water treated plants, but longer than Volutella minima treated plants, wherein lesion length was $0.5 \mathrm{~cm}$. 
Table 4

Effect of volatiles from saprobe fungi on colony diameter, mycelial growth rate (MGR), number and total weight of sclerotia of Sclerotinia sclerotiorum

\begin{tabular}{|c|c|c|c|c|}
\hline Treatments & $\begin{array}{c}\text { Colony diameter } \\
\text { at } 3^{\text {rd day }(\mathbf{c m})}\end{array}$ & $\begin{array}{c}\text { MGR } \\
(\mathrm{cm} / \text { day })^{\mathrm{c}}\end{array}$ & $\begin{array}{c}\text { Number of } \\
\text { sclerotia }\end{array}$ & $\begin{array}{c}\text { Total weight of } \\
\text { sclerotia (g) }\end{array}$ \\
\hline Control treatment ${ }^{\mathrm{a}}$ & $8.1 \pm 0.46 \mathrm{a}$ & $2.6 \pm 0.15 \mathrm{a}$ & $15.4 \pm 3.48 \mathrm{a}$ & $0.18 \pm 0.05^{\mathrm{n} . \mathrm{s}}$ \\
\hline Dictyosporium tetraseriale & $7.5 \pm 0.42 \mathrm{a}$ & $2.5 \pm 0.14 \mathrm{a}$ & $10.6 \pm 2.39 \mathrm{~b}$ & $0.14 \pm 0.04$ \\
\hline Myrothecium sp. isolate 2 & $7.7 \pm 0.43 \mathrm{a}$ & $2.6 \pm 015 \mathrm{a}$ & $12.4 \pm 2.80 \mathrm{a}$ & $0.12 \pm 0.03$ \\
\hline Phialomyces macrosporus & $3.1 \pm 0.17 \mathrm{~b}$ & $1.1 \pm 0.06 \mathrm{~b}$ & $8.8 \pm 1.99 b$ & $0.08 \pm 0.02$ \\
\hline Volutella minima & $8.0 \pm 0.45 \mathrm{a}$ & $2.6 \pm 0.15 \mathrm{a}$ & $8.2 \pm 1.85 \mathrm{~b}$ & $0.13 \pm 0.03$ \\
\hline Control treatment ${ }^{\mathrm{b}}$ & $7.9 \pm 0.45 \mathrm{a}$ & $2.5 \pm 0.14 \mathrm{a}$ & $15.4 \pm 3.55 \mathrm{a}$ & $0.19 \pm 0.05^{\mathrm{n} . \mathrm{s}}$ \\
\hline Dictyosporium tetraseriale & $7.8 \pm 0.44 \mathrm{a}$ & $2.6 \pm 0.14 \mathrm{a}$ & $11.2 \pm 2.58 \mathrm{~b}$ & $0.12 \pm 0.03$ \\
\hline Myrothecium sp. isolate 2 & $7.9 \pm 0.45 \mathrm{a}$ & $2.6 \pm 0.14 \mathrm{a}$ & $12.3 \pm 2.83 \mathrm{a}$ & $0.12 \pm 0.03$ \\
\hline Phialomyces macrosporus & $3.2 \pm 0.18 b$ & $1.2 \pm 0.07 \mathrm{~b}$ & $9.1 \pm 2.10 \mathrm{~b}$ & $0.07 \pm 0.02$ \\
\hline Volutella minima & $8.0 \pm 0.45 \mathrm{a}$ & $2.6 \pm 0.14 \mathrm{a}$ & $8.0 \pm 1.84 \mathrm{~b}$ & $0.13 \pm 0.03$ \\
\hline
\end{tabular}

${ }^{a}$ First experiment, mean \pm SE $(n=5$ replicates) within each column followed by the same letter are not significantly different $(\mathrm{P}<0.05)$ according to Tukey test. Not significant $(\mathrm{ns})$.

${ }^{b}$ Second experiment, mean \pm SE ( $n=5$ replicates) within each column followed by the same letter are not significantly different $(\mathrm{P}<0.05)$ according to Tukey test. Not significant $(\mathrm{ns})$.

${ }^{\mathrm{c}} \mathrm{MGR}=($ current colony diameter - colony diameter of the previous day $) /($ number of days after inoculation) $)$.

Table 5

Effect of volatiles from saprobe fungi on the germination of ascospores of Sclerotinia sclerotiorum

\begin{tabular}{lccc}
\hline Treatment & Germination (\%) & Germination inhibition (\%) \\
\hline Control treatment $^{\mathrm{a}}$ & $96.0 \pm 3.48$ & $\mathrm{a}$ & - \\
Dictyosporium tetraseriale & $92.6 \pm 3.35$ & $\mathrm{a}$ & 3.50 \\
Myrothecium sp. isolate 2 & $84.6 \pm 3.06$ & $\mathrm{a}$ & 11.9 \\
Phialomyces macrosporus & $67.6 \pm 2.45$ & $\mathrm{~b}$ & 29.6 \\
Volutella minima & $85.6 \pm 3.10$ & $\mathrm{a}$ & 10.8 \\
\hline Control treatment $^{\mathrm{b}}$ & $96.8 \pm 3.72$ & $\mathrm{a}$ & - \\
Dictyosporium tetraseriale & $92.6 \pm 3.56$ & $\mathrm{a}$ & 3.50 \\
Myrothecium sp. isolate 2 & $84.9 \pm 3.27$ & $\mathrm{a}$ & 11.9 \\
Phialomyces macrosporus & $67.8 \pm 2.61$ & $\mathrm{~b}$ & 29.6 \\
Volutella minima & $86.5 \pm 3.33$ & $\mathrm{a}$ & 10.8 \\
\hline
\end{tabular}

${ }^{a}$ First experiment, mean \pm SE $(n=5$ replicates) within each column followed by the same letter are not significantly different $(\mathrm{P}<0.05)$ according to Tukey test.

${ }^{b}$ Second experiment, mean \pm SE ( $n=5$ replicates) within each column followed by the same letter are not significantly different $(\mathrm{P}<0.05)$ according to Tukey test. 
Table 6

White mold lesion length at 7, 14 and 21 days after inoculation (DAI) and area under the disease progress curve (AUDPC) in soybean plants treated with water, acibenzolar-S-methyl (ASM) or saprobe fungi and inoculated with Sclerotinia sclerotiorum, under greenhouse conditions. Londrina-PR. Brazil. 2014

\begin{tabular}{lcccccc}
\hline \multirow{2}{*}{ Treatments } & \multicolumn{3}{c}{ Lesion length (cm) } & \multicolumn{2}{c}{ AUDPC } & $\begin{array}{c}\text { \% reduction } \\
\text { of AUDPC }\end{array}$ \\
\cline { 2 - 5 } & 7 DAI & 14 DAI & 21 DAI & & - \\
Control (water) ${ }^{\mathrm{a}}$ & $5.3 \pm 0.08 \mathrm{a}$ & $7.4 \pm 0.09 \mathrm{a}$ & $10.0 \pm 0.08 \mathrm{a}$ & $66.1 \pm 0.47 \mathrm{a}$ & 3.47 \\
ASM & $5.3 \pm 0.08 \mathrm{a}$ & $6.9 \pm 0.08 \mathrm{~b}$ & $9.5 \pm 0.08 \mathrm{~b}$ & $63.8 \pm 0.46 \mathrm{~b}$ & 1.82 \\
Dictyosporium tetraseriale & $5.3 \pm 0.08 \mathrm{a}$ & $7.1 \pm 0.08 \mathrm{~b}$ & $9.8 \pm 0.08 \mathrm{ab}$ & $64.9 \pm 0.46 \mathrm{ab}$ & 55.8 \\
Myrothecium sp. isolate 2 & $0.8 \pm 0.01 \mathrm{~b}$ & $6.1 \pm 0.07 \mathrm{c}$ & $9.7 \pm 0.08 \mathrm{ab}$ & $29.2 \pm 0.21 \mathrm{c}$ & 79.7 \\
Phialomyces macrosporus & $0.8 \pm 0.01 \mathrm{~b}$ & $2.1 \pm 0.02 \mathrm{~d}$ & $3.2 \pm 0.03 \mathrm{c}$ & $13.4 \pm 0.10 \mathrm{~d}$ & 83.2 \\
Volutella minima & $0.5 \pm 0.01 \mathrm{c}$ & $1.9 \pm 0.02 \mathrm{~d}$ & $3.1 \pm 0.02 \mathrm{c}$ & $11.1 \pm 0.08 \mathrm{e}$ & 8 \\
\hline
\end{tabular}

${ }^{a}$ Mean \pm SE ( $n=5$ replicates) within each column followed by the same letter are not significantly different $(\mathrm{P}<0.05)$ according to Tukey test.

${ }^{\mathrm{b}} \sum_{i=1}^{n}=\frac{\left[\left(X_{i}+X_{i+1}\right) / 2\right]\left[t_{i+1}-t_{i}\right]}{\left(T_{n}-T_{\mathrm{I}}\right)}$ where $X_{i}$ and $X_{i+1}$ are two consecutive severity measurements performed at times $t_{i}$ and $t_{i+1}$. respectively.

At 14 DAI all treatments showed lesion lengths lower than the negative control. Plants treated with ASM and D. tetraseriale exhibited lesion lengths of 6.9 and $7.1 \mathrm{~cm}$, respectively, both shorter than the negative control, but longer than Myrothecium sp. isolate 2 treated plants $(6.1 \mathrm{~cm})$. Plants treated with $P$. macrosporus and $V$. minima showed lesion length of 2.1 and $1.9 \mathrm{~cm}$, respectively, the shortest lesions recorded.

At 21 DAI, plants treated with D. tetraseriale and Myrothecium sp. showed lesion lengths not statistically different from the negative control and ASM treatment. Plants treated with P. macrosporus and $V$. minima with lesions of 3.2 and $3.1 \mathrm{~cm}$, respectively, showed the highest reduction of white mold severity.

Considering the area under the disease progress curve (AUDPC), the treatment with $D$. tetraseriale was the only one that was not statistically different from negative control. Plants treated with ASM showed shorter lesions from those of the control and the same length of the lesions of $D$. tetraseriale treated plants. Plants treated with $V$. minima showed the greatest reduction of disease progress, followed by plants treated with $P$. macrosporus and Myrothecium sp. isolate 2.

\section{Discussion}

Most saprobe filtrates showed antagonism against Sclerotinia sclerotiorum. Of the 25 saprobe fungi assessed, 72\% (18) reduced the MGR and $92 \%$ (22) reduced the number of sclerotia of $S$. sclerotiorum when tested at $50 \%$ concentration. Four saprobes completely inhibited the formation of sclerotia.

Abdullah, Ali and Suleman (2008) observed that Bacillus amyloliquefaciens filtrates $(50 \% \mathrm{v} / \mathrm{v})$ inhibited about $55 \%$ of the mycelial growth of $S$. sclerotiorum and reduced in 50\% the formation of sclerotia. Ávila et al. (2005) observed that two isolates of Trichoderma completely inhibited the colony growth of Sclerotium rolfsii. An in vitro study performed by Zancan, Machado, Sousa and Matos (2012), assessed the effect of fungicides and a biological product based on Trichoderma harzianum on the formation of sclerotia by $S$. sclerotiorum. Their results showed that methyl thiophanate and fluazinam completely inhibited sclerotia formation at concentrations above $100 \mathrm{ppm}$ and the biological control agent completely inhibited sclerotia formation at all tested concentrations. Figueirêdo et al. (2010) observed $37.04 \%$ and $32.94 \%$ reduction in severity of white mold of common bean using 
an isolate of T. harzianum and methyl thiophanate fungicide, respectively. Isolates of $T$. asperellum reduced apothecia density of $S$. sclerotiorum and white mold severity in the common bean, increasing the number of pods per plant and bean production by more than $40 \%$ (Geraldine et al., 2013). The T-aloe isolate of T. harzianum exhibited biocontrol potential of $S$. sclerotiorum, assessed by in vitro inhibition of mycelial growth, possible mycoparasitism, increase of activity of peroxidase, superoxide dismutase and catalase, reduction of the injury caused by S. sclerotiorum on soybean leaf cell membrane, as well as promotion of soybean plant growth and increase of chlorophyll and total phenol content. Also, the defense-related genes $P R 1, P R 2$, and $P R 3$ were expressed in the leaves of T-aloe-treated plants (Zhang et al., 2016).

Barros et al. (2015) performed a study to select fungal isolates with antagonistic effects on $S$. sclerotiorum and observed that Myrothecium sp. filtrate showed the greater inhibition potential in a dual culture assay. When soybean plants were treated with this fungus and inoculated 3 days after with $S$. sclerotiorum, the lesion length of white mold was reduced by $70 \%$ at 21 days after inoculation compared with inoculated plants without fungal filtrate treatment. Zheng et al. (2011), searched among 105 fungal isolates for new biocontrol agents for Verticillium dahliae Kleb. (Glomerellales: Plectosphaerellaceae) in cotton and observed that one belonging to the genus Myrothecium showed effectiveness of $33.21 \%$ under greenhouse conditions.

The reduction on ascospore germination by Myrothecium sp. isolate 2 was the same as the chemical control fluazinam. Sclerotinia sclerotiorum develops apothecia from sclerotia followed by production of ascospores, which can be disseminated at short distances (Abawi \& Grogan, 1979) and the infection in soybean plant in the field is primarily through ascospores infecting flower petals (Grau \& Hartman, 2015). Therefore, products that reduce ascospore germination are important in controlling white mold in field conditions, as these structures are the only source of airborne inoculum of this disease. Sumida et al. (2015) verified under field conditions that procymidone and fluazinam fungicides (combined with benzalkonium chloride or alone), reduced the incidence of Sclerotinia stem rot in about $74 \%$.

The inhibitive effect of volatile compounds from $P$. macrosporus on the mycelial growth and ascospore germination of $S$. sclerotiorum assessed in our experiments was reported too on the mycelial growth and sporulation of Colletotrichum gloeosporioides (Penz.) Penz., Sacc. (Glomerellales: Glomerellaceae) (Rodríguez et al., 2016) and on the bacterial pathogen Pseudomonas syringae pv. garcae (Botrel, 2013).

Organic volatile compounds (OVCs) are substances of low polarity and molecular weight that can easily transpose membranes and be released in the atmosphere and soil (Pichersky, Noel, \& Dudareva, 2006). Many microorganisms produce a mixture of OVCs that may be toxic to beneficial and phytopathogenic fungi (Morath, Hung, \& Bennet, 2012; Kottb, Gigolashvili, Großkinsky \& Piechulla, 2015) by reducing mycelial growth, sporulation and germination (Li et al., 2012; Morath et al., 2012). Due to their efficient spread through soil porosity, organic volatile compounds could be an alternative strategy to control soil inhabitant fungi (Dudareva, Negre, Nagegowda, \& Orlova, 2006).

Despite the aggressive inoculation methodology used in the experiment, soybean plants treated preventively with $V$. minima, $P$. macrosporus and Myrothecium sp. isolate 2 filtrates exhibited significantly lower disease severity during the 21 days of evaluation. Our results of white mold control with ASM suggest that this commercial chemical inducer does not efficiently control this disease in soybean plants and confirmed previous results on the same pathosystem (Barros et al., 2015). 
The direct antagonistic activity of P. macrosporus filtrates and volatiles against $S$. sclerotiorum verified in our in vitro experiments could be complemented by a resistance induction mechanism already found in other pathosystems. Phialomyces macrosporus is reported in literature as a potential resistance inducer in coffee plants against Pseudomonas syringae pv. garcae (Botrel et al., 2018) and Colletotrichum gloeosporioides (Rodríguez et al., 2016) and in eucalyptus plants against Puccinia psidii Winter (Pucciniales: Pucciniaceae) (Pierozzi, 2013).

The in vivo experiment was carried out with inoculation of soybean plants with $S$. sclerotiorum mycelium, and therefore, reflects the ability of the tested biocontrol agents to inhibit mycelial growth. In this context, $P$. macrosporus and $V$. minima filtrates have a higher inhibitory effect. The high efficacy of Myrothecium sp. isolate 2 to inhibit ascospore germination was not assessed in our in vivo experiment due to the inoculation method used. New experiments should be performed with inoculation of plants with ascospore suspension to test the potential of Myrothecium sp. isolate 2 in controlling white mold derived from ascospore infection.

Collectively, our results underline the efficacy of Myrothecium sp. isolate 2, P. macrosporus and $V$. minima as biocontrol agents of soybean white mold. Further studies are needed to determine if there is a resistance induction mechanism being activated in soybean plants when the filtrates are sprayed prior to pathogen inoculation. Finally, studies are essential to determine how viable the use of these saprobe fungi is under field conditions, including aspects such as the optimal saprobe formulation, timing, and number of saprobe sprays.

\section{Acknowledgments}

The first author was the recipient of a MSc. scholarship (N. 1194680) and the third author received a post-doctoral scholarship (N. 1615283) both from CAPES (Coordination for the Improvement of Higher Education Personnel). The project had financial support N. 563380/20101 from CNPQ (Brazilian National Council for Scientific and Technological Development) and N. 2010/52343-0 from FAPESP (The São Paulo Research Foundation).

\section{References}

Abawi, G., \& Grogan, R. (1979). Epidemiology of diseases caused by Sclerotinia species. Phytopathology, 69(8), 899-904. doi: 10.1094/Phyto-69-899

Abdullah, M. T., Ali, N. Y., \& Suleman, P. (2008). Biological control of Sclerotinia sclerotiorum (Lib.) de with Trichoderma harzianum and Bacillus amyloliquefaciens. Crop Protection, 27(10), 13541359. doi: 10.1016/j.cropro.2008.05.007

Almeida, A. M. R., Ferreira, L. P., Yorinori, J. T., Silva, J. F. V., Henning, A. A., Godoy, C. V., \& Costamilan, L. M. M. M. (2005). Doenças da soja (Glycine max). In L. Amorim, Jorge, A. M. R., \& Bergamin, A. $\mathrm{F}^{\circ}$. (Eds.), Manual de fitopatologia (4a ed., pp. 569588). São Paulo: Editora Agronômica Ceres, BR.

Almeida, D. A. C., Izabel, T. S. S., \& Gusmão, L. F. P. (2011). Fungos conidiais do bioma Caatinga I. Novos registros para o continente americano. Rodriguésia, 62(1), 43-53. doi: 10.1590/2175-7860201162104

Ávila, Z. R., Carvalho, S. S., Braúna, L. M., Gomes, D. M. P. A., Silva, M. C. F., \& Mello, S. C. M. M. (2005). Seleção de isolados de Trichoderma spp. antagônicos a Sclerotium rolfsii e Sclerotinia sclerotiorum. (Boletim Técnico. 2005). Brasilia, DF: EMBRAPA Recursos Genéticos.

Barbosa, F. R., \& Gusmão, L. F. P. (2011). Conidial fungi from semi-arid Caatinga Biome of Brazil. Rare freshwater hyphomycetes and other new records. Mycosphere, 2(4), 475-485. Recuperado de http:// mycosphere.org/pdfs/MC2_4_No10.pdf

Bardin, S. D., \& Huang, H. C. (2001). Research on biology and control of Sclerotinia diseases in Canada ${ }^{1}$. Canadian Journal Plant Pathology, 23(1), 88-98. doi: 10.1080/07060660109506914

Barros, D. C. M., Fonseca, I. C. B., Balbi-Peña, M. I., Pascholati, S. F. \& Peitl, D. C. (2015). Biocontrol of Sclerotinia sclerotiorum and white mold of soybean using saprobic fungi from semi-arid areas of Northeastern Brazil. Summa Phytopathologica, 41(4), 251-255. doi: 10.1590/0100-5405/2086 
Boland, G. J., \& Hall, R. (1994). Index of plant hosts of Sclerotinia sclerotiorum. Canadian Journal Plant Pathology, 16(2), 93-108. doi: 10.1080/07060669409500766

Botrel, D. A. (2013). Fungos sapróbios como agentes de biocontrole da mancha aureolada do cafeeiro causada por Pseudomonas syringae pv. garcae. Dissertação de mestrado, Universidade Federal de Lavras, Lavras, MG, Brasil.

Botrel, D. A., Laborde, M. C. F., Ferreira, M. C., Medeiros, F. H. V., Resende, M. L. V., Ribeiro, P. M., Jr.,... Gusmão, L. F. P. (2018). Saprobic fungi as biocontrol agents of halo blight (Pseudomonas syringae pv. garcae) in coffee clones. Coffee Science, 13(3), 283-291. doi: 10.25186/cs.v13i3.1438

Chun, D., Kao, L. B., Lockwood, J. L., \& Isleib, T. G. (1987). Laboratory and field assessment of resistance in soybean to stem rot caused by Sclerotinia sclerotiorum. Plant Disease, 71(9), 811-815. doi: 10.1094/ PD-71-0811

Danielson, G. A., Nelson, B. D., \& Helms, T. C. (2004). Effect of sclerotinia stem rot on yield of soybean inoculated at different growth stages. Plant Disease, 88(3), 297-300. doi: 10.1094/PDIS.2004.88.3.297

Dhingra, O. D., Mendonça, H. L., \& Macedo, D. M. (2009). Doenças e seu controle. Tecnologia de produção e usos da soja. Londrina: Mecenas.

Dudareva, N., Negre, F., Nagegowda, D. A., \& Orlova, I. (2006). Plant volatiles: recent advances and future perspectives. Critical Reviews Plant Sciences, 25(5), 417-440. doi: 10.1080/07352680600899973

Elias, L. M., Domingues, M. V. P. F., Moura, K. E., Harakava, R., \& Patricio, F. R. A. (2016). Selection of Trichoderma isolates for biological control of Sclerotinia minor and S. sclerotiorum in lettuce. Summa Phytopatholica, 42(3), 216-221. doi: 10.1590/0100-5405/2147

Figueirêdo, G. S., Figueirêdo, L. C., Cavalcanti, F. C. N., Santos, A. C., Costa, A. F., \& Oliveira, N. T. (2010). Biological and chemical control of Sclerotinia sclerotiorum using Trichoderma spp. and Ulocladium atrum and pathogenicity to bean plants. Brazilian Archives of Biology and Technology, 53(1), 1-9. doi: 10.1590/S1516-89132010000100001

Fry, W. E. (1978). Quantification of general resistance of potato cultivars and fungicide effects for integrated control of potato late blight [caused by Phytophthora infestans]. Phytopathology, 68(11), 1650-1655. Recuperado de https://www.apsnet.org/publications/ phytopathology/backissues/Documents/1978 Articles/Phyto68n11 1650.PDF
Geraldine, A. M., Lopes, F. A. C., Carvalho, D. D. C., Barbosa, E. T., Rodrigues, A. R., Brandão, R. S.,... Lobo, M., Jr. (2013). Cell wall-degrading enzymes and parasitism of sclerotia are key factors on field biocontrol of white mold by Trichoderma spp. Biological Control, 67(3), 308-316. doi: 10.1016/j. biocontrol.2013.09.013

Grau, C. R., \& Hartman, G. L. (2015). Sclerotinia stem rot. In: G. L. Hartman, J. C. Rupe, E. J. Sikora, L. L. Domier, J. A. Davis, \& K. L. Steffey (Eds.), Compendium of soybean diseases and pests (pp. 5962). St. Paul: American Phytopathological Society.

Hoffman, D. D., Hartman, G. L., Mueller, D. S., Leitz, R. A., Nichkell, C. D., \& Pedersen, W. L. (1998). Yield and seed quality of soybean cultivars infected with Sclerotinia sclerotiorum. Plant Disease, 82(7), 826829. doi: 10.1094/PDIS.1998.82.7.826

Kamal, M. M., Lindbeck, K. D., Savocchia, S., \& Ash, G. J. (2015). Biological control of sclerotinia stem rot of canola using antagonistic bacteria. Plant Pathology, 64(6), 1375-1384. doi: 10.1111/ppa.12369

Kottb, M., Gigolashvili, T., Großkinsky, D. K., \& Piechulla, B. (2015). Trichoderma volatiles effecting Arabidopsis: from inhibition to protection against phytopathogenic fungi. Frontiers in Microbiology, (6), 995. doi: 10.3389/fmicb.2015.00995

Kull, L. S., Vuong, T. D., Powers, K. S., Eskridge, K. M., Steadman, J. R., \& Hartman, G. L. (2003). Evluation of resistance screening methods for sclerotinia stem rot of soybean and dry bean. Plant Disease, 87(12), 1471-1476. doi: 10.1094/PDIS.2003.87.12.1471

Leão-Ferreira, S. M., Pascholati, S. F., Gusmão. L. F., \& Castañeda Ruiz, R. F. (2013). Conidial fungi from the semi-arid Caatinga biome of Brazil. Three new species and new records. Nova Hedwigia, 96(3-4), 479-494. doi: 10.1127/0029-5035/2013/0084

Li, Q., Ning, P., Zheng, L., Huang, J., Li, G., \& Hsiang, T. (2012). Effects of volatile substances of Streptomyces globisporus JK-1 on control of Botrytis cinerea on tomato fruit. Biological Control, 61(2), 113-120. doi: 10.1016/J.BIOCONTROL.2011.10.014

McCredie, T. A., \& Sivasithamparam, K. (1985). Fungi mycoparasitic on sclerotia of Sclerotinia sclerotiorum in some. Western Australian soils. Transactions of the British Mycological Society, 84(4), 736-739. doi: 10.1016/S0007-1536(85)80133-9

Morath, S. U., Hung, R., \& Bennett, J. W. (2012). Fungal volatile organic compounds: a review with emphasis on their biotechnological potential. Fungal Biology Reviews, 26(2-3), 73-83. doi: 10.1016/J. FBR.2012.07.001 
Morton, J. G., \& Hall, R. (1989). Factors determining the efficacy of chemical control of white mold in white bean. Canadian Journal Plant Pathology, 11(3), 297-302. doi: 10.1080/07060668909501116

Nicot, P. C., Avril, F., Duffaud, M., Leyronas, C., Troulet, C., Villeneuve, F., \& Bardin, M. (2019). Differential susceptibility to the mycoparasite Paraphaeosphaeria minitans among Sclerotinia sclerotiorum isolates. Tropical Plant Pathology, 44(1), 82-93. doi: 10.1007/s40858-018-0256-7

Oliveira, J. A. (1991). Efeito do tratamento fungicida em sementes e no controle de tombamento de plântulas de pepino (Cucumis sativus L.) e pimentão (Capsicum annum L.). Tese de doutorado, Universidade Federal de Lavras. Lavras, MG, Brasil.

Peitl, D. C., Araujo, F. A., Gonçalves, R. M., Santiago, D. C., Sumida, C. H., \& Balbi-Peña, M. I. (2017). Biological control of bacterial spot of tomato by saprobe fungi from semi-arid areas of northeastern Brazil. Semina: Ciências Agrárias, 38(3), 12511263. doi: 10.5433/1679-0359.2017v38n3p1251

Pichersky, E., Noel, J. P., \& Dudareva, N. (2006). Biosynthesis of plant volatiles: nature's diversity and ingenuity. Science, 311(5762), 808-811. doi: 10.1126/science. 1118510

Pierozzi, C. G. (2013). Fungos sapróbios do semiárido nordestino: aspectos fisiológicos, ação no controle da ferrugem e indução de enraizamento em mudas de eucalipto. Dissertação de mestrado, Universidade Estadual Paulista, Faculdade de Ciências Agronômicas, Botucatu, SP, Brasil.

Rabeendran, N., Jones, E. E., Moot, D. J., \& Stewart, A. (2006). Biocontrol of Sclerotinia lettuce drop by Coniothyrium minitans and Trichoderma hamatum. Biological Control, 39(3), 352-362. doi: 10.1016/J. BIOCONTROL.2006.06.004

Resende, R. S., Milagres, C. A., Rezende, D., Aucique-Perez, C. E., \& Rodrigues, F. A. (2015). Bioprospecting of saprobe fungi from the semi-arid north-east of Brazil for the control of anthracnose on sorghum. Journal of Phytopathology, 163(10), 787794. doi: 10.1111/jph. 12376

Ribeiro, A. I., Costa, E. S., Thomasi, S. S., Brandão, D. F. R., Vieira, P. C., Fernandes, J. B.,... Silva, M. F. G. F. (2018). Biological and chemical control of Sclerotinia sclerotiorum using Stachybotrys levispora and its secondary metabolite griseofulvin. Journal Agriculture and Food Chemistry, 66(29), 7627-7632. doi: 10.1021/acs.jafc.7b04197
Rodríguez, G. A. A., Abreu, M. S., Pinto, F. A. M. F., Monteiro, A. C. A., Núñez, Á. M. P., Resende, M. L. V.,... Medeiros, F. H. V. (2016). Phialomyces macrosporus decreases anthracnose severity on coffee seedlings by competition for nutrients and induced resistance. Biological Control, 103, 19-128. doi: 10. 1016/J.BIOCONTROL.2016.08.009

Santa Izabel, T. D. S., \& Gusmão, L. F. P. (2018). Richness and diversity of conidial fungi associated with plant debris in three enclaves of Atlantic Forest in the Caatinga biome of Brazil. Plant Ecology and Evolution, 151(1), 35-47. doi: 10.5091/ plecevo.2018.1332

Sarma, B. K., Ameer Basha, S., Singh, D. P., \& Singh, U. P. (2007). Use of non-conventional chemicals as an alternative approach to protect chickpea (Cicer arietinum) from Sclerotinia stem rot. Crop Protection, 26(7), 1042-1048. doi: 10.1016/j. cropro.2006.09.015

Shaner, G., \& Finney, R. E. (1977). The effect of nitrogen fertilization on the expression of slow-mildewing resistance in Knox wheat. Phytopathology, 67(8), 1051-1056. doi: 10.1094/Phyto-67-1051.

Sumida, C. H., Canteri, M. G., Peitl, D. C., Tibolla, F., Orsini, I. P., Araújo, F. A.,... Calvos, N. S. (2015). Chemical and biological control of Sclerotinia stem rot in the soybean crop. Ciência Rural, 45(5), 760766. doi: 10.1590/0103-8478cr20140198

Sumida, C. H., Daniel, J. F. S., Araujo, A. P. C. S., Peitl, D. C., Abreu, L. M., Dekker, R. F. H., \& Canteri, M. G. (2018). Trichoderma asperelloides antagonism to nine Sclerotinia sclerotiorum strains and biological control of white mold disease in soybean plants. Biocontrol Science and Technology, 28(2), 142-156. doi: 10.1080/09583157.2018.1430743

Sun, P., \& Yang, X. B. (2000). Light, temperature, and moisture effects on apothecium production of Sclerotinia sclerotiorum. Plant Disease, 84(12), 1287-1293. doi: 10.1094/PDIS.2000.84.12.1287

Tolêdo-Souza, E. D., \& Costa, J. L. S. (2007). Métodos de inoculação de plântulas de feijoeiro para avaliação de germoplasma quanto a resistência a Sclerotinia sclerotiorum (Lib.) De Bary. Pesquisa Agropecuária Tropical, 33(32), 57-63. Recuperado de https://www. revistas.ufg.br/pat/article/view/ 2348/2330

United States Department of Agriculture (2020). World agricultural supply and demand estimates. Retrieved from https://www.usda.gov/oce/commodity/wasde/ 
Vinodkumar, S., Nakkeeran, S., Renukadevi, P., \& Malathi, V. G. (2017). Biocontrol potentials of antimicrobial peptide producing bacillus species: multifaceted antagonists for the management of stem rot of carnation caused by Sclerotinia sclerotiorum. Frontiers in Microbiology, 8, 446. doi: 10.3389/ fmicb.2017.00446

Whipps, J. M., Sreenivasaprasad, S., Muthumeenakshi, S., Rogers, C. W., \& Challen, M. P. (2008). Use of Coniothyrium minitans as a biocontrol agent and some molecular aspects of sclerotial mycoparasitism. European Journal of Plant Pathology, 121(3), 323330. doi: 10.1007/s10658-007-9238-1

Yang, F., Abdelnabby, H., \& Xiao, Y. (2015). A mutant of the nematophagous fungus Paecilomyces lilacinus (Thom) is a novel biocontrol agent for Sclerotinia sclerotiorum. Microbial Pathogenesis, 89, 169-176. doi: 10.1016/j.micpath.2015.10.012

Zancan, A. W. L., Machado, J. C., Sousa, B. F. M., \& Matos, C. S. M. (2012). Mycelial growth, production and germination of sclerotia of Sclerotinia sclerotiorum in the presence of fungicides and Trichoderma harzianum. Bioscience Journal, 28(5), 782-789. Recuperado de http://www.seer.ufu.br/ index.php/ biosciencejournal/article/view/13909.
Zeng, W., Wang, D., Kirk, W., \& Hao, J. (2012). Use of Coniothyrium minitans and other microorganisms for reducing Sclerotinia sclerotiorum. Biological Control. 60(2), 225-232. doi: 10.1016/J. BIOCONTROL.2011.10.009

Zhang, F., Ge, H., Zhang, F., Guo, N., Wang, Y., Chen, L.,... Li, C. (2016). Biocontrol potential of Trichoderma harzianum isolate T-aloe against Sclerotinia sclerotiorum in soybean. Plant Physiology and Biochemistry, 100, 64-74. doi:10.1016/j. plaphy.2015.12.017

Zheng, Y., Xue, Q.-Y., Xu, L.-L., Xu, Q., Lu, S., Gu, C., \& Guo, J.-H. (2011) A screening strategy of fungal biocontrol agents towards Verticillium wilt of cotton. Biological Control, 56(3), 209-216. doi: 10.1016/ J.BIOCONTROL.2010.11.010 
